\title{
Oscillations in the Developing Cortex: A Mechanism for Establishing and Synchronizing an Early Network?
}

\author{
Abhishek Banerjee ${ }^{1 \star}$ and Tommas J. Ellender ${ }^{2 \star}$ \\ ${ }^{1}$ Neuronal Oscillations Group, Department of Physiology, Anatomy and Genetics, University of Oxford, Oxford OX1 3PT, United Kingdom, and ${ }^{2}$ Medical \\ Research Council Anatomical Neuropharmacology Unit, University of Oxford, Oxford OX1 3TH, United Kingdom \\ Review of Yang et al.
}

Neural circuit organization during early postnatal development requires both patterned gene expression and synchronized electrical activity ("oscillations"), which shape the sensory structures in the cortex (Rakic, 1988; Katz and Shatz, 1996; Spitzer, 2006). This rhythmic electrical activity has been suggested to be instrumental in controlling neuronal differentiation, synaptogenesis, and synaptic plasticity (Khazipov and Luhmann, 2006). The spatiotemporal dynamics of these oscillations in vivo, as well as the role of peripheral sensory input in generating these patterns, are not well understood.

A recent study by Yang et al. (2009) in The Journal of Neuroscience examined the spatiotemporal dynamics, developmental profile, and mechanisms of these early developmental oscillations in vivo in the rat somatosensory cortex (S1). The authors performed long-lasting extracellular multielectrode recordings from all cortical layers simultaneously, using an array of $4 \times 4$ electrodes with $200 \mu \mathrm{m}$ spacing, in neonatal rats at different ages ranging from postnatal day (P)0 to P7. They reported the presence of three distinct forms of synchronized oscillatory activity, namely, spindle bursts $(\sim 10$

Received Sept. 15, 2009; revised 0ct. 19, 2009; accepted 0ct. 23, 2009.

A.B. holds a Felix scholarship. T.J.E. holds a Medical Research Council postdoctoral fellowship.

${ }^{*}$ A.B. and T.J.E. contributed equally to this work.

Correspondence should be addressed to Abhishek Banerjee, Department of Physiology, Anatomy and Genetics, University of Oxford, Parks Road, 0xford 0X13PT, UK. E-mail: abhishek.banerjee@dpag.ox.ac.uk. DOI:10.1523/JNEUROSCI.4567-09.2009

Copyright $\odot 2009$ Society for Neuroscience $\quad$ 0270-6474/09/2915029-02\$15.00/0
$\mathrm{Hz})$, gamma oscillations $(\sim 40 \mathrm{~Hz})$, and long oscillations $(\sim 12 \mathrm{~Hz})$.

Consistent with previous work (Khazipov et al., 2004; Dupont et al., 2006), Yang et al. (2009) found that spindle bursts (short second long bursts of $\sim 10 \mathrm{~Hz}$ oscillations) represented the dominant activity pattern in S1, with the majority of events occurring synchronously at recording sites spaced $\sim 200-400 \mu \mathrm{m}$ horizontally and $\sim 600 \mu \mathrm{m}$ vertically, resembling the dimensions of a cortical column. The spindle bursts were found in all animals recorded and showed an age-dependent increase in occurrence (from two to six times per minute), amplitude (121 to $442 \mu \mathrm{V})$, and intrinsic frequency ( 8 to $11 \mathrm{~Hz}$ ) during the first postnatal week.

Whereas spindle bursts had been observed previously, this article describes for the first time the presence of fast gamma oscillations and long oscillations in the cortex of young rat pups. Gamma oscillations (200 ms events of $\sim 40 \mathrm{~Hz}$ oscillations) were confined to the barrel field and were more localized than spindle bursts $(\sim 200 \times$ $\sim 200 \mu \mathrm{m}$ ) and showed a similar developmental increase in occurrence (from two to seven times per minute) and amplitude (95 to $154 \mu \mathrm{V}$ ) but their intrinsic frequency remained constant. The third subtype, long oscillations, occurred infrequently, once every $20 \mathrm{~min}$, and often had a duration of $>50 \mathrm{~s}$. Of all three types of oscillations they were the most widespread and could be recorded from all areas of S1 synchronously $(\sim 600 \times \sim 600 \mu \mathrm{m})$. They resemble $\mathrm{Ca}^{2+}$ waves in their spatial synchronization and might play a role in regulating long-distance wiring in the immature cortex (Garaschuk et al., 2000; Peinado, 2000).

Unlike spindle bursts, the gamma and long oscillations occurred in only $\sim 50 \%$ of the animals. The absence of these oscillations in some animals, possibly due to gender, age, or hormonal differences, may be worth investigating in future experiments. Additionally, because the type of anesthetics used and the depth of anesthesia often affect the occurrence and the characteristics of observed oscillations in vivo, these factors may also have been responsible for the absence of oscillations in a subset of animals. Interestingly, the authors found that the characteristics of oscillations were the same when deep ice cooling was used for anesthesia, supporting the idea that these are physiologically relevant activity patterns and not due to a pharmacological effect secondary to anesthetic administration.

The authors further investigated whether these oscillations are generated intrinsically in $\mathrm{S} 1$ and, if not, the extent to which peripheral sensory input plays a role in their initiation. By stimulating the whisker pad both mechanically and electrically, Yang et al. (2009) found that they could induce spindle bursts as well as gamma oscillations with properties identical to the ones occurring spontaneously. Conversely, silencing of peripheral sensory input, by injecting $2 \%$ lidocaine subcutaneously into the whisker pad, significantly reduced both spindle bursts 
and gamma oscillations. The already low incidence of long oscillations under control conditions precluded any similar conclusion being drawn, and if investigated would involve very long recording times. These results suggest that peripheral sensory input can drive oscillations in $\mathrm{S} 1$ and that oscillations can occur without whisker input also, but it is unknown whether the latter are generated intrinsically in S1 or are the result of input from other brain regions.

Assuming that different types of oscillations have different functions during cortical development, further investigation of which type of input from the whisker pad leads to an induced spindle burst or gamma oscillation would be interesting. In addition, the extent to which the intrinsically generated cortical oscillations are dependent on early sensory input remains unknown. Detailed investigation using prolonged sensory deprivation (e.g., slow and extended lidocaine release from implanted polymer beads or depriving a row of whiskers) would further clarify the role of sensory input in shaping intrinsic cortical activity. Recently, it has been observed that different sensory deprivation paradigms, i.e., monocular eyelid closure or drug-induced retinal inactivation, can have major consequences for the properties of individual neurons and by extension that of the network and network activity (Linden et al., 2009).

During early development, thalamocortical axons grow and interact with subplate neurons before they synapse onto layer 4 neurons within the cortical plate. The transient subplate neuron population plays an important role in thalamocortical axon pathfinding during early stages of neural circuit refinement (Katz and Shatz, 1996; Catalano and Shatz, 1998). Furthermore, in vitro studies have shown that the subplate is essential in driving oscillations in the early somatosensory cortical network, which switches from an electrically activated, gapjunction-coupled, subplate-driven syncitium to an NMDA-receptor-dependent chemical synaptic network (Dupont et al., 2006). To investigate the role of subplate in the generation of oscillations in vivo, Yang et al. (2009) used current-source density analysis on events recorded from all cortical layers simultaneously. They found a sink in the subplate concurrent with a source in the cortical plate in $\sim 60-70 \%$ of cases, for all three types of oscillations. In addition, the multiunit activity in the subplate seemed to precede that of the cortex. These results suggest that activity is driven from the subplate, and subplate ablation experiments using immunotoxins could confirm their direct involvement. However, the subplate most likely consists of a heterogeneous population of both excitatory and inhibitory neurons with different morphophysiological properties (Allendoerfer and Shatz, 1994). Recent techniques, such as optogenetic activation of specific populations of subplate neurons, would be better suited to determine how specific subplate neuron types are involved in generating early network oscillations. The remaining $30-40 \%$ of the cortical oscillations were without a significant sink in the subplate, suggesting local cortical generation, but these might have been driven by activity arriving from other cortical areas or hemisphere.

As it has been shown that both NMDA receptors and gap junctions are important for oscillations in vitro (Dupont et al., 2006), the authors investigated whether these could also play a role in generating oscillations in vivo. Topical application of the NMDA receptor antagonist DL-AP5 or the gap junction blocker carbenoxolone to the cortical surface significantly reduced spindle and gamma oscillations by $\sim 50 \%$, suggesting their involvement in the generation of these oscillations. However, because the authors did not apply both blockers simultaneously, it is not known whether they are necessary and sufficient for the generation of spindle and gamma oscillations. Further investigation of the role of gap junctions in the generation of these oscillations could additionally be investigated in juvenile Connexin $36^{-1-}$ mice.

Layer 4 of somatosensory cortex also contains a gap-junction-coupled glial network (Houades et al., 2008). Gliotransmission modulates slow cortical oscillations in the adult somatosensory cortex (Fellin et al., 2009) and might also have an important role in the generation of oscillations seen during development. Furthermore, GABA, which has been shown to be depolarizing during the first postnatal week (Ben-Ari, 2002), might contribute to the early network activity observed. A cortical circuit consisting of only excitatory neurons, with an intrinsic resonance frequency at $\sim 40 \mathrm{~Hz}$, could also generate gamma oscillations (Pike et al., 2000). The relationship between single cells and network activity could be further investigated by combining multielectrode recordings with in vivo patching of individual cortical neurons.

In summary, this interesting study shows for the first time the existence of several distinct types of oscillatory activity in the developing somatosensory cortex in vivo. These oscillations have varying developmental and spatiotemporal properties and, most likely, have different functions in the development of the cortical circuitry. The cellular mechanisms underlying each type of oscillation are only beginning to be elucidated and will provide much insight into their potential role in cortical development. Future work will help us to understand the interaction between these oscillations and patterned gene expression in laying the foundations of the cortical template and later shaping and refining the cortical circuitry.

\section{References}

Allendoerfer KL, Shatz CJ (1994) The subplate, a transient neocortical structure: its role in the development of connections between thalamus and cortex. Annu Rev Neurosci 17:185-218.

Ben-Ari Y (2002) Excitatory actions of gaba during development: the nature of the nurture. Nat Rev Neurosci 3:728-739.

Catalano SM, Shatz CJ (1998) Activity-dependent cortical target selection by thalamic axons. Science 281:559-562.

Dupont E, Hanganu IL, Kilb W, Hirsch S, Luhmann HJ (2006) Rapid developmental switch in the mechanisms driving early cortical columnar networks. Nature 439:79-83.

Fellin T, Halassa MM, Terunuma M, Succol F, Takano H, Frank M, Moss SJ, Haydon PG (2009) Endogenous nonneuronal modulators of synaptic transmission control cortical slow oscillations in vivo. Proc Natl Acad Sci U S A 106:15037-15042.

Garaschuk O, Linn J, Eilers J, Konnerth A (2000) Large-scale oscillatory calcium waves in the immature cortex. Nat Neurosci 3:452-459.

Houades V, Koulakoff A, Ezan P, Seif I, Giaume C (2008) Gap junction-mediated astrocytic networks in the mouse barrel cortex. J Neurosci 20:5207-5217.

Katz LC, Shatz CJ (1996) Synaptic activity and the construction of cortical circuits. Science 274:1133-1138.

Khazipov R, Luhmann HJ (2006) Early patterns of electrical activity in the developing cerebral cortex of humans and rodents. Trends Neurosci 29:414-418.

Khazipov R, Sirota A, Leinekugel X, Holmes GL, Ben-Ari Y, Buzsáki G (2004) Early motor activity drives spindle bursts in the developing somatosensory cortex. Nature 432:758-761.

Linden ML, Heynen AJ, Haslinger RH, Bear MF (2009) Thalamic activity that drives visual cortical plasticity. Nat Neurosci 4:390-392.

Peinado A (2000) Traveling slow waves of neural activity: a novel form of network activity in developing neocortex. J Neurosci 20:RC54.

Pike FG, Goddard RS, Suckling JM, Ganter P, Kasthuri N, Paulsen O (2000) Distinct frequency preferences of different types of rat hippocampal neurones in response to oscillatory input currents. J Physiol 529 Pt 1:205-213.

Rakic P (1988) Specification of cerebral cortical areas. Science 241:170-176.

Spitzer NC (2006) Electrical activity in early neuronal development. Nature 444:707-712.

Yang JW, Hanganu-Opatz IL, Sun JJ, Luhmann HJ (2009) Three patterns of oscillatory activity differentially synchronize developing neocortical networks in vivo. J Neurosci 29:9011-9025. 